\title{
Caractérisation thermo-physique des matériaux thermo-dégradables
}

\author{
Adrien Bouvet ${ }^{1,2, a}$, Didier Demange ${ }^{1}$, Philippe Herve $^{2}$ et Rachid Bennacer ${ }^{3}$ \\ 1 ONERA - DMSC, Fort de Palaiseau, Chemin de la Hunière, 91761 Palaiseau, Cedex, France \\ 2 LEEE, Laboratoire d'énergétique et d'économie d'énergie, Université Paris X Nanterre, 1 chemin Desvalliére, \\ 92410 Ville d'Avray, France \\ 3 LEEVAM/LEEE, Laboratoire d'énergétique environnement valorisation matériaux, Université Cergy Pontoise, \\ 5 mail Gay Lussac, 95031 Neuville sur Oise, France
}

Résumé - L'objectif de l'étude est de caractériser thermiquement des nouveaux matériaux, utilisés dans les protections thermiques : les matériaux thermo-dégradables. À cet effet, des moyens de mesure répondant aux spécificités, intrinsèques et d'utilisations, des matériaux thermo-dégradables ont été développés par l'ONERA.

Mots clés : Matériau thermo-dégradable / caractérisation thermo-physique / thermogravimétrie / conductivité thermique / diffusivité thermique

\begin{abstract}
The aim of the study is to characterise the thermal properties of new materials, used in thermal protections: the degradable materials. To this end, specifically measurement techniques answering to the intrinsic characteristic, of these materials were developed by ONERA.
\end{abstract}

Key words: Thermo-degradable material / thermo-physical characterisation / thermogravimetry / thermal conductivity / thermal diffusivity

\begin{tabular}{|llll|}
\hline \multicolumn{2}{l}{ Nomenclature } & & \\
$\lambda$ & conductivité thermique, $\mathrm{W} \cdot \mathrm{m}^{-1} \cdot \mathrm{K}^{-1}$ & $Q$ & énergie, $\mathrm{J}$ \\
$\lambda_{\mathrm{G}}$ & conductivité thermique globale & $k$ & constante d'étalonnage \\
$\lambda_{\mathrm{l}}$ & conductivité thermique locale & $m$ & masse, $\mathrm{kg}$ \\
$\varepsilon$ & émissivité & $m_{\mathrm{f}}$ & masse finale, $\mathrm{kg}$ \\
$\alpha$ & absorption & $m_{0}$ & masse initiale, $\mathrm{kg}$ \\
$a$ & diffusivité thermique, $\mathrm{m}^{2} \cdot \mathrm{s}^{-1}$ & $e$ & épaisseur de l'échantillon \\
$\rho$ & masse volumique, $\mathrm{kg} \cdot \mathrm{m}^{-3}$ & $A, B, n$ & constantes Arrhenius \\
$C p$ & chaleur spécifique, J.kg $\mathrm{kg}^{-1} \cdot \mathrm{K}^{-1}$ & $R$ & constante molaire des gaz \\
$T$ & température, $\mathrm{K}$ & $i$ & indice de l'état du matériau \\
$\Delta U$ & différence de potentiel, $\mathrm{V}$ & & \\
\hline
\end{tabular}

\section{Introduction}

De nombreuses applications nécessitent l'utilisation de barrières thermiques. Bien qu'elles aient toutes le même objectif, limiter l'influence des flux de chaleur, il en existe

a Auteur correspondant : Bouvet@onera.fr différents types. Les barrières thermiques peuvent être regroupées en deux grandes catégories celles dites « inertes » et celles dites « réactives »

Dans cette étude, nous nous intéressons aux matériaux thermo-dégradables constituant certaines barrières thermiques dites réactives. Ces matériaux, comme leur nom l'indique, sont uniquement utilisées pour des applications à structure consommable. 
Les applications peuvent être civiles, spatiales utilisés pour satelliser une charge en orbite, ou militaires, domaines dans lesquels ils sont déjà utilisés comme protections thermiques des moteurs de missile.

L'intérêt porté à ces matériaux est dû à deux phénomènes :

- le caractère endothermique de la réaction de thermodégradation permettant de dissiper de l'énergie.

- la phase gazeuse issue de la réaction jouant le rôle de fluide caloporteur qui contribue à évacuer de l'énergie.

Le but de cette étude est de caractériser les matériaux thermo-dégradables de façon à pouvoir, par la suite, réaliser des simulations numériques sur une protection thermique complète.

\section{Les matériaux thermo-dégradables}

Les matériaux thermo-dégradables sont des matériaux très intéressants du point de vue thermique.

Effectivement, les réactions chimiques, aux caractères endothermiques, couplées à l'écoulement gazeux, induit par la thermo-dégradation, leurs confèrent une capacité d'isolation transitoire particulièrement élevée, plus efficace qu'une isolation inerte. De plus, la thermodégradation entraîne une diminution de la masse ce qui est, par exemple, en parfaite adéquation avec les applications aéronautiques de façon à augmenter la vitesse et la portée des engins.

En revanche, l'étude de ces matériaux est complexe. En effet, les réactions de thermo-dégradation n'étant pas réversibles, leurs propriétés thermiques sont fortement non-linéaires. Elles sont inévitablement liées à la température et à l'avancement de la réaction. L'avancement de la réaction est lui-même lié à l'historique en température (effet mémoire) et à la vitesse de chauffe. De plus, les gammes de température d'utilisation et de vitesse de chauffe sont variées; elles sont respectivement de 300 à $2500 \mathrm{~K}$ pour la température et de 0,1 à $100 \mathrm{~K} . \mathrm{s}^{-1}$ à l'allumage d'un moteur dans le cas d'une application aéronautique.

Dans ce contexte, la caractérisation expérimentale des propriétés thermiques, pour tous les stades de la transformation chimique et pour tous les niveaux de température concernés, est impossible. Néanmoins, sur certains domaines de température le matériau est quasiment inerte (Fig. 1).

La figure 1 montre l'évolution de la masse volumique d'un matériau thermo-dégradable. Cette évolution est celle d'une silicone chargée de poudre de carbone. La ligne continue foncée représente la masse volumique en fonction de la température. Les traits discontinus horizontaux représentent respectivement les masses volumiques des trois états pseudo stables. La masse volumique initiale du matériau est nommée $\rho_{1}$, celle de l'état intermédiaire $\rho_{2}$ et celle de l'etat final $\rho_{3}$.

On peut constater que cette silicone subit deux phases de réaction chimique distinctes, soit trois états

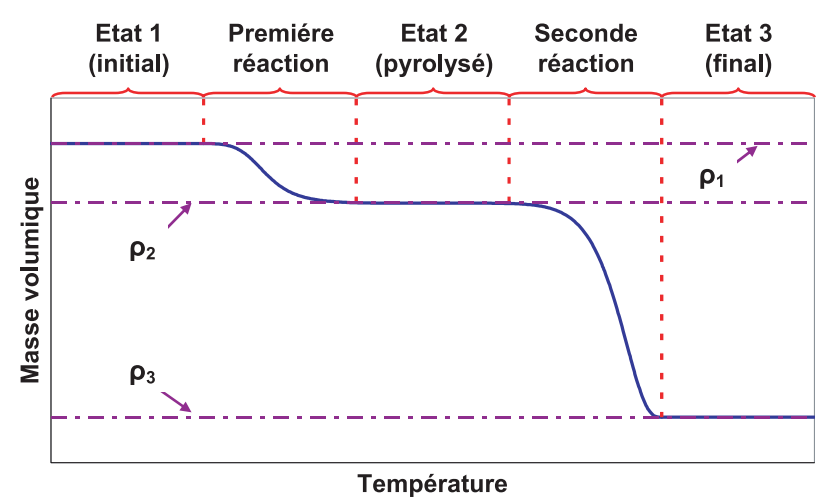

Fig. 1. Evolution de la masse volumique d'un matériau thermo-dégradable subissant deux pertes de masse distinctes

structuraux, au cours d'une élévation en température. Il est important de préciser que chaque phase de réaction peut être composée de plusieurs réactions élémentaires.

La détermination des paramètres thermiques $(\lambda, \mathrm{Cp})$ est alors réalisée en plusieurs étapes.

Premièrement l'étude macroscopique de la cinétique des réactions chimiques, par thermogravimétrie, permet de déterminer les domaines de température sur lesquels le matériau est stable.

Deuxièmement, les paramètres thermiques sont mesurés sur chaque zone de stabilité par une méthode classique.

Enfin, pour les températures correspondant à une phase de réaction, les propriétés sont calculées à partir, de la masse volumique et des valeurs issues des états stables encadrant la réaction.

La modélisation des structures utilisant les matériaux thermo-dégradables nécessite la connaissance de nombreux paramètres thermo-physiques. Dans cet article nous nous intéresserons aux propriétés suivantes :

- l'évolution de la masse volumique, soit la cinétique des réactions chimiques,

- l'évolution de la conductivité thermique,

- l'évolution de la diffusivité thermique,

- l'évolution de la capacité calorifique de la partie solide (à titre de vérification),

- la chaleur spécifique des gaz de dégradation.

Deux autres propriétés importantes peuvent être citées :

- l'enthalpie de réaction, elle peut être mesurée par ACD (Analyse Calorimétrique Différentielle) [1],

- la perméabilité et la porosité des matériaux. Ces propriétés structurales peuvent faire l'objet d'une étude afin de mieux caractériser les phénomènes d'évacuation des gaz de dégradation au travers de la structure.

\section{Dispositifs expérimentaux}

Dans le domaine des mesures des propriétés thermiques : diffusivité thermique, conductivité thermique et chaleur spécifique, les moyens existants, utilisés pour 
des matériaux inertes, doivent être modifiés afin de tenir compte des propriétés propres aux matériaux thermodégradables (réactions de dégradations) ainsi que de leurs conditions d'utilisation extrêmes (températures élevées $2800 \mathrm{~K}$ avec des vitesses de chauffe pouvant atteindre $\left.100 \mathrm{~K} . \mathrm{s}^{-1}\right)$. Dans un premier temps, il est important de borner les domaines de température sur lesquels le matériau est stable et ceux sur lesquels les réactions ont lieu.

\subsection{Etude macroscopique de la cinétique des réactions chimiques}

La cinétique des réactions chimiques est étudiée via une analyse thermogravimétrique (ATG) rapide [2].

Cette méthode repose sur le principe que le produit de la réaction est constitué, au moins, d'une phase gazeuse. Dans ce cas, l'avancement de la réaction est supposé proportionnel à l'évolution de la masse de l'échantillon. Ainsi, il faut connaître la masse et la température de l'échantillon au cours du temps pour caractériser le processus de dégradation.

Pour ce faire, un banc de thermogravimétrie rapide a été mis en place. L'échantillon est placé au centre d'un élément chauffant cylindrique en graphite. La géométrie du résistor est optimisée pour obtenir des vitesses de chauffe élevées, représentatives des conditions réelles d'utilisations de ces matériaux (de 0,1 à $100 \mathrm{~K} . \mathrm{s}^{-1}$ ). Le processus de dégradation étant largement influencé par l'atmosphère ambiante l'ensemble est placé dans une enceinte fermée sous atmosphère contrôlée. Lorsque les essais doivent être réalisés sous atmosphère neutre, c'est généralement le cas étant donné les conditions d'utilisation de ces matériaux, il est préférable d'utiliser de l'hélium, afin d'assurer un transfert d'énergie efficace entre le résistor et l'échantillon notamment pour les températures modérées $(<1000 \mathrm{~K})$.

La mesure de la température directe de l'échantillon pose problème. Il est bien entendu impossible d'implanter un thermocouple dans l'échantillon à caractériser faute de perturber la mesure de la masse. Ainsi, la température est mesurée en deux points en dessous et au dessus de l'échantillon. Un premier thermocouple est implanté dans le résistor au dessus de l'échantillon et un second thermocouple est implanté dans un deuxième échantillon dit «échantillon témoin », identique au premier, placé sous l'échantillon à tester (Fig. 2).

Un second point important, pour déterminer la loi d'évolution de la masse en fonction de la température, la température au sein de l'échantillon doit être homogène.

Afin que la température soit la plus homogène possible la taille des échantillons testés est faible. Nous utilisons un cylindre creux de $10 \mathrm{~mm}$ de hauteur, $4 \mathrm{~mm}$ de diamètre extérieur et $2 \mathrm{~mm}$ de diamètre intérieur. En revanche, la petite taille des échantillons engendre une variation de masse très faible, inférieure à $100 \mathrm{mg}$, et nécessite ainsi un moyen de pesée très sensible. La masse de l'échantillon est mesurée, au cours du temps, par une microbalance à fléau (Setaram), dont la sensibilité est inférieure à $1 \mu \mathrm{g}$.

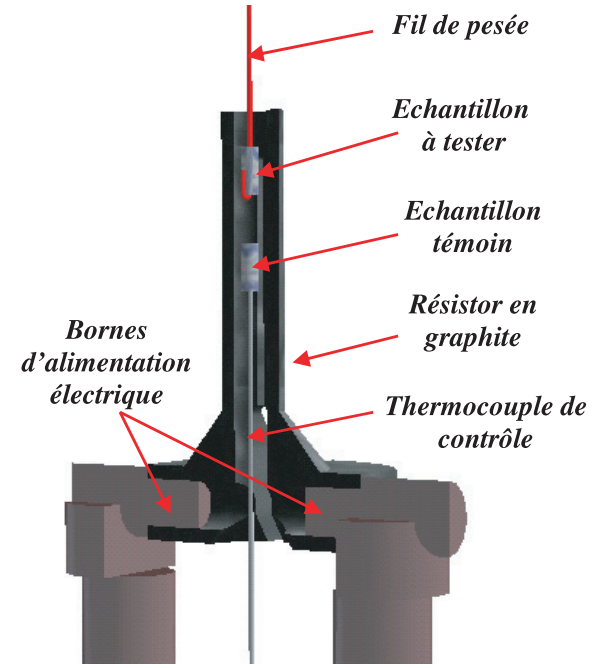

Fig. 2. Schéma du dispositif de thermogravimétrie rapide.

Le dispositif est par ailleurs couplé à un spectromètre infrarouge permettant d'analyser les gaz émis en continu. De plus, les gaz émis sont collectés au cours de l'essai. Ainsi, à la fin de l'essai, ils peuvent être analysés par un chromatographe en phase gazeuse ou par un spectromètre de masse. L'analyse des gaz émis permet de connaître sa composition chimique et ainsi d'estimer son pouvoir calorifique.

A la fin d'un essai de thermogravimétrie, l'évolution de la masse, donc de la masse volumique, en fonction de la température et de la vitesse de chauffe est connue (Fig. 1). Et, connaissant la composition du gaz de dégradation, nous pouvons en déduire la capacité calorifique du gaz. Afin que le matériau soit totalement décrit thermiquement, il faut, en plus de la masse volumique, caractériser deux des trois paramètres suivants, la conductivité thermique, la diffusivité thermique ou la capacité calorifique du matériau.

\subsection{Mesure de la conductivité thermique}

Par définition une mesure directe de la conductivité thermique nécessite d'introduire un gradient de température dans le matériau. Par conséquent, les mesures ne peuvent pas être effectuées à une température donnée c'est à dire à un taux de dégradation homogène. Dans un premier temps, l'objectif est de caractériser les états stables du matériau. Pour ce faire, deux conductimètres complémentaires ont été développés (chauffage par contact et sans contact). Les deux dispositifs sont basés sur le principe de la face chaude imposée. Cette méthode consiste, à imposer une température sur l'une des faces d'un échantillon plan, et à mesurer d'une part le flux thermique qui traverse l'éprouvette et d'autre part la température de la face opposée lorsque le régime permanent est établi. Ainsi, la loi de Fourier permet de déterminer la conductivité thermique. 


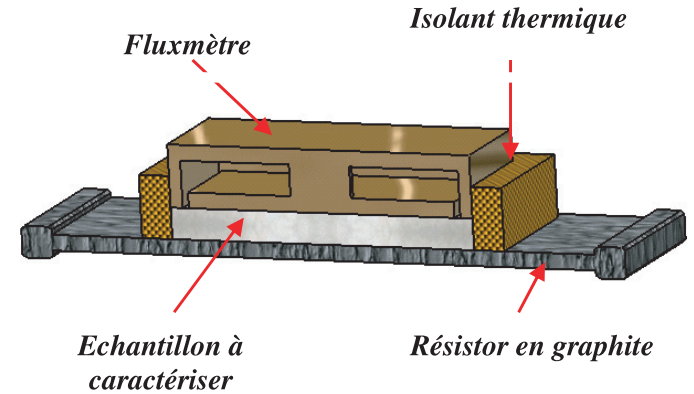

Fig. 3. Schéma du conductimètre, utilisant un résistor en graphite comme source de chauffage.

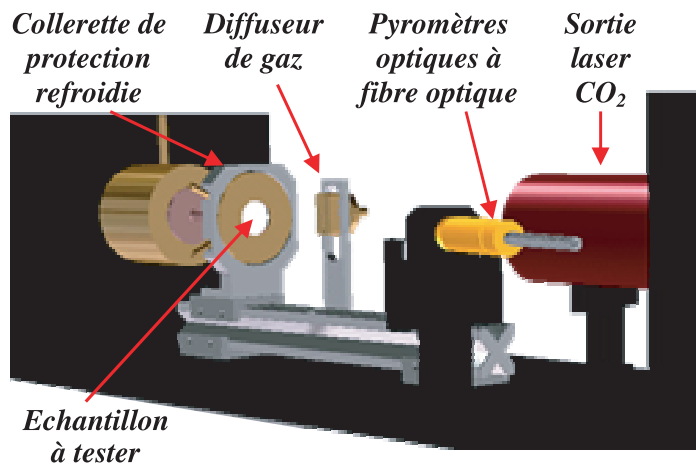

Fig. 4. Schéma du conductimètre, utilisant un laser $\mathrm{CO} 2$ comme source de chauffage.

- Le conductimètre utilisant un chauffage par contact Ce dispositif est composé d'une enceinte fermée sous atmosphère contrôlée. Le chauffage est assuré par le contact de l'échantillon avec la face d'un résistor en graphite (Fig. 3). Ce conductimètre est parfaitement adapté aux matériaux très isolants $\left(0,01\right.$ à $\left.1,0 \mathrm{~W} \cdot \mathrm{m}^{-1} \cdot \mathrm{K}^{-1}\right)$. En revanche, lorsque le matériau se déforme, même très légèrement, la résistance de contact résistor/matériaux augmente fortement et fausse totalement la mesure de conductivité. Or, la taille conséquente des échantillons entraîne inévitablement une légère déformation. Cette méthode est donc utilisée pour caractériser les matériaux à l'état vierge $[3,4]$.

- Le conductimètre utilisant un chauffage par Laser

Ce dispositif utilise un laser $\mathrm{CO}_{2}$ continu haute puissance comme source de chauffage de façon à contourner le problème du contact imparfait rencontré par la première méthode (Fig. 4). La température en face chaude est contrôlée par trois pyromètres optiques dont un proche ultra-violet afin de minimiser l'influence du facteur d'émissivité spectrale sur le calcul de la température dite vraie. Le récepteur thermique, en face froide, est constitué de deux thermocouples et d'un fluxmètre à gradient associé à un calorimètre. Ce dispositif permet de caractériser des matériaux présentant une conductivité comprise entre 0,5 et $100 \mathrm{~W} \cdot \mathrm{m}^{-1} \cdot \mathrm{K}^{-1}$ sur une large gamme de température ( $700 \mathrm{~K}$ à $2800 \mathrm{~K}$ ).

A priori, à ce niveau de la caractérisation, la connaissance de la diffusivité thermique ou de la capacité calorifique permettrait de connaître l'ensemble des propriétés

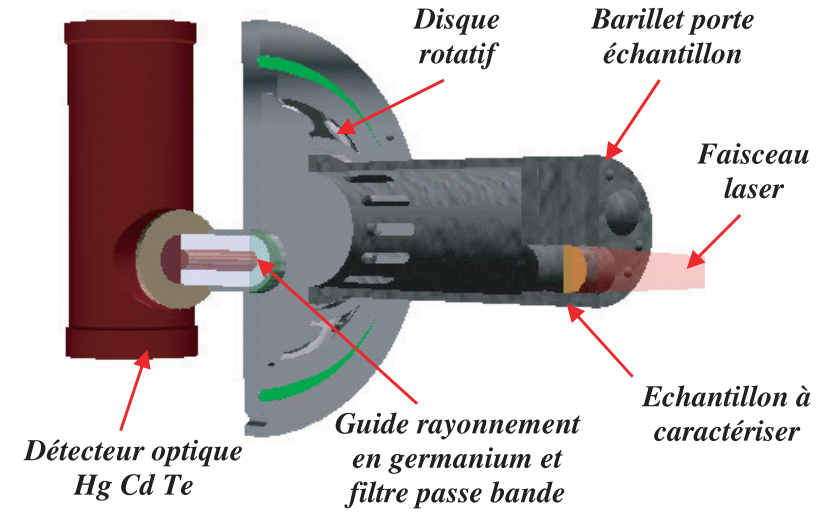

Fig. 5. Schéma du diffusimètre utilisant un laser $\mathrm{CO}_{2}$ comme source impulsionnelle.

thermiques du matériau via la définition de la diffusivité thermique. Néanmoins, étant donné les hypothèses qui ont été émises, il est préférable de mesurer les deux paramètres afin de s'assurer de l'exactitude des résultats.

\subsection{Mesure de la diffusivité par la méthode "flash" et estimation simultanée de la capacité calorifique}

La méthode de mesure de la diffusivité thermique la plus couramment utilisée est la méthode flash et notamment face arrière. Dans son principe, cette méthode impulsionnelle consiste à soumettre la face avant d'un échantillon plan à une impulsion de flux de chaleur de courte durée, devant les phénomènes de propagation, et à observer l'évolution temporelle de la température en face arrière. La vitesse d'homogénéisation de la température, au sein de l'éprouvette, est liée à la diffusivité thermique. Ainsi, pour une épaisseur donnée la mesure de la diffusivité consiste à mesurer un temps à un stade donné du processus d'homogénéisation de la température $[4,5]$.

L'échantillon est placé dans un four en graphite, sous atmosphère contrôlée. L'élévation de température en face arrière est mesurée par un détecteur $\mathrm{HgCdTe}$ refroidi à l'azote liquide (Fig. 5). Ce dispositif autorise les mesures sur une large gamme de température, de la température ambiante à des températures élevées $2800 \mathrm{~K}$, tout en conservant une détectivité suffisante.

Le diffusimétre développé présente plusieurs particularités. La première réside dans l'utilisation d'un laser $\mathrm{CO} 2$ continu, couplé à un obturateur, comme source impulsionnelle. Ce dispositif permet de générer des impulsions relativement longues (quelques centièmes de secondes) de faible puissance. Ainsi, l'énergie délivrée est suffisante pour caractériser des matériaux très isolants, même à haute température, sans pour autant détériorer la structure de la face avant par une augmentation locale de la température trop importante. De plus, la longueur d'onde du rayonnement laser émis $(10,6 \mu \mathrm{m})$ permet même de caractériser les matériaux à base de céramique qui à haute température demeurent généralement opaques à cette longueur d'onde. 
Une seconde particularité; le porte échantillons est rotatif et il contient trois échantillons. Ce dispositif permet d'introduire un échantillon témoin afin d'obtenir une estimation de la capacité calorifique des deux autres matériaux testés en diffusivité. Pour ce faire, le faisceau laser est divisé, une partie vient éclairer l'échantillon et l'autre partie est dirigée sur un calorimètre. Ainsi, connaissant l'énergie relative reçue par l'échantillon et l'élévation de température relative de l'échantillon par identification avec l'échantillon référence nous pouvons en déduire la capacité calorifique.

\section{Méthodes de dépouillement et résultats obtenus}

\subsection{Dépouillement d'un essai thermogravimétrique}

En fin d'essai, l'évolution de la masse en fonction de la température est connue. Ainsi, les différents états de la structure sont connus et bornés. La perte de masse peut, à priori, être modélisée par une loi d'Arrhenius.

$$
\frac{\partial m}{\partial t}=-m_{0} \cdot A \cdot e^{-\frac{B}{R \cdot T}} \cdot\left(\frac{m-m_{f}}{m_{0}}\right)^{n}
$$

Effectivement, à une vitesse de chauffe donnée, l'utilisation d'une loi d'Arrhenius aux coefficients constants procure une estimation satisfaisante de la loi de pertes de masse. La différence constatée est généralement inférieure à $5 \%$. En revanche, lorsque la vitesse de chauffe du processus à modéliser varie fortement, par exemple la barrière thermique d'une chambre de combustion à l'allumage du moteur, cette modélisation est trop simplifiée. Dans ce cas, il est préférable d'adapter les coefficients de la loi d'Arrhenius en fonction de la température (Fig. 6) [4,5].

La figure 6 illustre la perte de masse d'une silicone chargée de poudre de carbone au cours de quatre élévations de températures à différentes vitesses de chauffe. L'influence de la vitesse de chauffe est largement mise en évidence, plus la vitesse de chauffe augmente plus la température d'activation de la réaction est élevée. Les valeurs mesurées (courbes continues) sont approchées (losanges) avec une loi d'Arrhenius aux coefficients variables en fonction de la vitesse de chauffe.

\subsection{Dépouillement d'un essai de conductivité}

En fin d'essai, les paramètres mesurés et enregistrés, quel que soit le dispositif utilisé, sont les suivants :

- la température de la face chaude en deux points (au bord et au centre de l'échantillon),

- la température de la face froide en deux points (au bord et au centre de l'échantillon),

- le flux en face arrière.

La conductivité expérimentale globale, sous fort gradient thermique, est calculée via la loi de Fourier. Effectivement, une vitesse de chauffe lente, adaptée à chaque

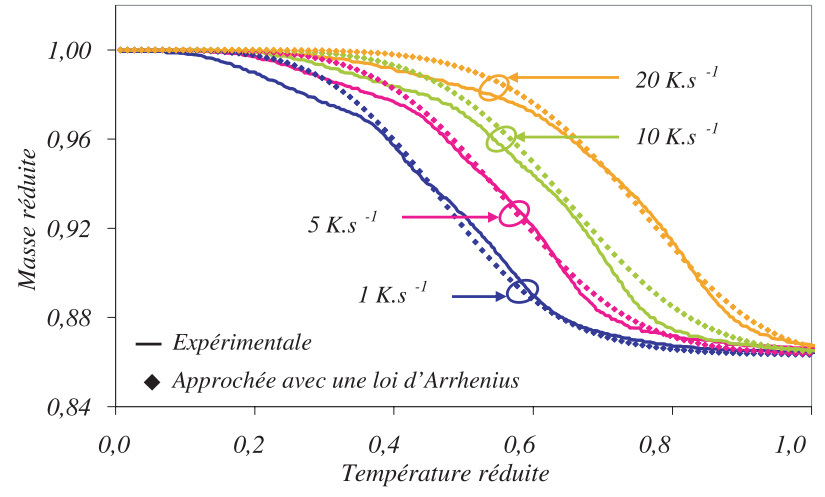

Fig. 6. Evolution de la masse d'un matériau thermodégradable mesurée et approchée avec une loi d'Arrhenius.

matériau en fonction de sa conductivité, permet de négliger le terme instationnaire. Ainsi, le calcul de la conductivité globale est immédiat, elle est obtenue par la relation suivante :

$$
\lambda_{G}^{\dagger}(t)=\frac{\varphi(t) \cdot e}{T_{C}(t)-T_{F}(t)}
$$

Le processus est répété sur chaque zone de stabilité. Dans les zones où le matériau est dans un état

$$
C p_{\text {mat }}=k_{\text {étalon }} \cdot \frac{\alpha_{\text {mat }} \cdot \varepsilon_{\text {mat }}}{m_{\text {mat }}} \cdot\left(\frac{\Delta U_{1}}{\Delta U_{2}}\right)_{\text {mat }}
$$

intermédiaire, une régression linéaire, à partir de la masse volumique courante et des conductivités des deux états de stabilité encadrant la réaction, permet d'estimer la conductivité thermique locale pour n'importe quel état d'avancement de la réaction et pour tous les niveaux de température [6]. Pour la zone réactionnelle entre l'état 1 et l'état 2 la conductivité thermique est donnée par :

$$
\lambda(T)=\lambda_{1}(T)-\left(\lambda_{1}(T)-\lambda_{2}(T)\right) \frac{\left(\rho_{1}-\rho\right)}{\left(\rho_{1}-\rho_{2}\right)}
$$

La figure 7, ci-dessous, est composée de deux graphiques. Le graphique du haut reprend l'évolution de la masse volumique en fonction de la température pour une élévation de la température à $0,5 \mathrm{~K} . \mathrm{s}^{-1}$. Sur la deuxième partie du graphique les lignes continues représentent les conductivités thermique des états stables (réellement mesurées), la ligne en pointillés est reconstruite via l'interpolation linéaire (3).

L'incertitude sur $\lambda_{l}$, issue de cette méthode, est inférieure à $15 \%$.

\subsection{Dépouillement d'un essai de diffusivité et estimation de la chaleur spécifique}

A la fin d'un essai de diffusivité, le thermogramme obtenu représente l'évolution de la température de la face arrière au cours du temps.

Le dépouillement d'un essai consiste alors à comparer la réponse expérimentale mesurée à une réponse théorique 


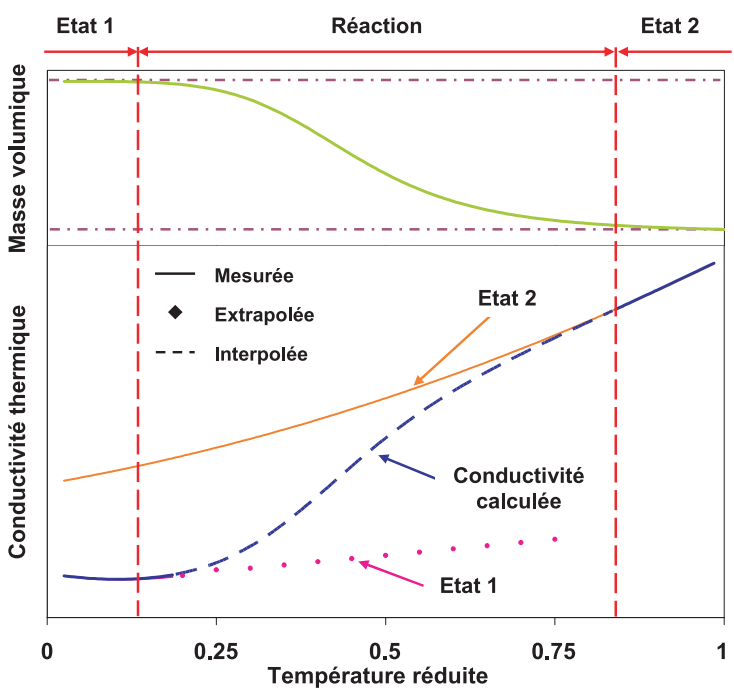

Fig. 7. Evolution de la conductivité thermique et de la masse volumique d'un matériau thermo-dégradable (pour $\mathrm{dT} / \mathrm{dt}=$ $\left.0,5 \mathrm{~K} . \mathrm{s}^{-1}\right)$.

calculée. Le thermogramme théorique est calculé à partir d'un modèle thermocinétique avec prise en compte des pertes thermiques [7-9].

Un calorimètre mesure l'énergie laser émise au cours de l'impulsion et par suite l'énergie $Q$ absorbée par l'échantillon est calculée via le coefficient d'absorption de l'échantillon. L'élévation de température relative $\Delta T$ est mesurée par le détecteur infrarouge. Les valeurs de $Q$ et de $\Delta T$ sont données par les relations suivantes :

$$
Q=\alpha \cdot k_{1} \cdot \Delta U_{1} \quad \Delta T=k_{2} \cdot \frac{\Delta U_{2}}{\varepsilon}
$$

Ainsi, l'obtention de l'équation qui détermine la capacité calorifique est donnée par :

Le coefficient $k_{\text {étalon, }}$ qui regroupe $k_{1}$ et $k_{2}$, est mesuré via un essai sur un échantillon référence tel que la zircone ou l'alumine. L'intérêt d'utiliser l'un de ces deux matériaux comme échantillon de référence est la connaissance de leur émissivité à la fréquence du détecteur $\mathrm{HgCdTe}$ couplé au filtre passe bande (fenêtre de $2 \mu \mathrm{m}$ centré à $8 \mu \mathrm{m}$ ) et de leur coefficient d'absorption à la fréquence du laser. De plus, la capacité calorifique de ces matériaux est bien connue [11]. $k_{\text {étalon }}$ est calculé via l'équation suivante :

$$
k_{\text {étalon }}=\frac{C p_{\text {ref }} \cdot m_{r e f}}{\alpha_{r e f} \cdot \varepsilon_{r e f}} \cdot\left(\frac{\Delta U_{2}}{\Delta U_{1}}\right)_{r e f}
$$

La figure 8 représente la capacité calorifique de l'alumine mesurée via cette méthode et celle couramment utilisé dans la littérature. Cette figure illustre clairement la précision des mesures sur une large gamme de température.

Comme pour la conductivité thermique, lorsque les états stables du matériau sont caractérisés, la capacité calorifique dans le domaine de réaction est calculée par une loi des mélanges [6]. Par exemple pour la zone

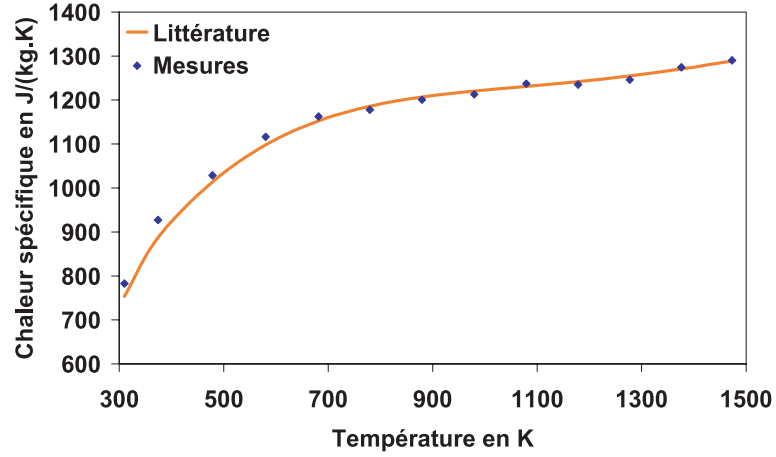

Fig. 8. Capacité calorifique d'une alumine mesurée à partir d'un essai de diffusivité (référence de Zircone)

réactionnelle entre l'état 1 et 2 la relation est la suivante :

$$
\frac{\rho \cdot C p(T)}{\rho_{1} \cdot C p_{1}(T)}=\frac{\rho_{2} \cdot C p_{2}(T)}{\rho_{1} \cdot C p_{1}(T)} \cdot \frac{\left(\rho_{1}-\rho\right)}{\left(\rho_{1}-\rho_{2}\right)}+\frac{\left(\rho-\rho_{2}\right)}{\left(\rho_{1}-\rho_{2}\right)}
$$

\section{Conclusion}

Les dispositifs expérimentaux présentés dans cet article ont permis de caractériser les propriétés thermo physiques (masse volumique, conductivité thermique, diffusivité thermique et capacité calorifique) de divers matériaux thermo-dégradables sur une large gamme de température. La combinaison de ces différentes approches permet une caractérisation continue sur la large gamme de température.

\section{Références}

[1] J.M. Diot, Capacités thermiques, Techniques de l'ingénieur, R 2970, Janvier 1993

[2] J.L. Daudon, Thermogravimétrie, Techniques de l'ingénieur, P 1260, Mars 2001

[3] D. Demange, Nouvelle méthode de caractérisation thermique des super-isolants, Congrès français de thermique, SFT 2002 édition Elsevier, pages 529-534

[4] G. Darmon, D. Balageas, Transfert de chaleur et de masse dans un matériau composite pyrolysable, revue générale de thermique $\mathrm{n}^{\circ}$ 292, Avril 1986, pages 197-206

[5] J.F. Epherre, L. Laborde, Pyrolysis of carbon phenolic composites, Fourth International Symposium Atmospheric re-entry vehicles and systems, 2005

[6] J.P. Lasserre, J.F. Epherre, V. Ducamp, Identification et modélisation de la conductivité thermique à haute température d'un composite carbone/résine pyrolysé, SFT 2001, pages 555-560

[7] D. Demange, New applications of the flash method, High Temperatures - High Pressures, 2002 Vol. 34, pages 9-18

[8] A. Degiovanni, Diffusivité et méthode flash, Revue Générale de Thermique ${ }^{\circ}{ }^{\circ} 185$, mai 1977 , pages $421-438$

[9] H.S. Carslaw and J.C. Jaeger, Conduction of heat in solids, Oxford university press, second edition 1959

[10] P. Hervé, Mesure de l'émissivité thermique, Techniques de l'ingénieur, R 2737, Septembre 2005

[11] NIST-National Institute of Standards and Technology http://webbook.nist.gov 